\title{
REYNOLDS STRESSES DERIVED FROM SIMULATIONS
}

\author{
P. PULKKINEN \\ Dept. of Theoretical Physics, P.O.Box 9, FIN-00014 University of Helsinki, Finland \\ I. TUOMINEN \\ Observatory and Astrophysics Laboratory, P.O.Box 14, FIN-00014 University of \\ Helsinki, Finland
}

A. BRANDENBURG

NORDITA, Blegdamsvej 17, DK-2100 Copenhagen $\varnothing$, Denmark

A. NORDLUND

Copenhagen University Observatory, Øster Voldgade 3, DK-1350 Copenhagen K, Denmark

and

R.F. STEIN

Dept. of Physics and Astronomy, Michigan State University, East Lansing, MI 48824, $U S A$

\begin{abstract}
Three-dimensional simulations of convection are studied with specific attention to the effects of rotation and latitude on different quantities. The latitude-dependence of the strength of convection is found to decrease with increasing strength of the turbulence. The $\Lambda$-effect is, however, still present also in a more turbulent case.
\end{abstract}

\section{Introduction}

We simulate convection in a rectangular rotating box assuming periodicity in the horizontal directions with stress-free upper and lower boundaries. We use a modified version of the code by Nordlund \& Stein (1989). Gravity points in the vertical $(z)$ direction but the rotation axis is tilted, describing the situation at some latitude away from the pole. The calculation starts from a polytropic atmosphere with velocity perturbations. Each layer's stability is governed by the polytropic index. For details of the system setup, see Pulkkinen et al. (1993). A typical run takes about 8 hours of Cray X-MP cpu-time, one time step lasting about 0.3 seconds for a grid of $31^{3}$ and 1.4 seconds for the a larger one of $63 \times 63 \times 37$.

We consider two different cases: case I, a single unstable layer and case II, an unstable layer above a stable layer, into which convective motions can penetrate. The physics of the system is described by dimensionless numbers, the most important of which are the Rayleigh number, the Taylor number, and the Prandtl number:

$$
\mathrm{Ra}=\left(\frac{g d^{4}}{\kappa \nu} \frac{1}{c_{p}} \frac{\mathrm{d} s}{\mathrm{~d} z}\right)_{z=z_{m}}, \quad \mathrm{Ta}=\frac{4 \Omega^{2} d^{4}}{\nu^{2}}, \quad \operatorname{Pr}=\frac{\nu}{\kappa} .
$$

In Table I we give values of some input parameters and typical values of a few quantities obtained from our simulations.

When simulating stellar convection, the main limitation is the restricted range of different time and length scales that can be included. For economical reasons we consider models with relatively small ratios of rotation period and sound travel 
Summary of the parameters in cases I and II. (The Prandtl number is in all cases unity, and $\mathrm{Ma}$ is the Mach number).

\begin{tabular}{lll}
\hline quantity & case I & case II \\
\hline & & \\
grid & $31 \times 31 \times 31$ & $63 \times 63 \times 37$ \\
$\mathrm{Ra}$ & $3 \times 10^{4}, 6 \times 10^{4}$ & $10^{8}$ \\
$\mathrm{Ta}$ & $10^{4}$ & $10^{7}$ \\
$\mathrm{Ma}$ & 0.03 & 0.1 \\
$\tau_{\text {rot }} / \tau_{\text {sound }}$ & 50 & 10 \\
$\mathrm{Ro}^{-1}$ & 3.3 & 3.2 \\
\hline
\end{tabular}

time. A more important quantity, however, is the inverse Rossby number, $\mathrm{Ro}^{-1}=$ $2 \Omega \tau_{\text {conv }}$, where $\tau_{\text {conv }}$ is the convective turnover time. In case II a rather small value for the radiative diffusion $\kappa$ was chosen in order to decrease the total flux input at the bottom. In order to stabilize the code an additional "hyperdiffusion" has been included in case. II. Although case II is closer to the real convection zone with respect to the higher Rayleigh and Taylor numbers it has a smaller separation between the time scales than case $\mathrm{I}$. This is because the largest contribution in increasing the Rayleigh and Taylor numbers goes to the entropy gradient and to angular velocity. Case II is, however, more turbulent than case I, and in that sense more realistic.

\section{Theoretical Background}

In order to investigate stellar differential rotation driven by turbulent convection one usually separates the different scales by dividing the velocity field $\mathbf{u}$ in two parts, $\mathbf{u}=\langle\mathbf{u}\rangle+\mathbf{u}^{\prime}$ where $\langle\mathbf{u}\rangle$ is the large-scale mean field and $\mathbf{u}^{\prime}$ is the small-scale fluctuation field. Inserting this in the hydrodynamic momentum equation produces, after taking a horizontal average over the whole equation, the Reynolds equation

$$
\rho\left(\frac{\partial\langle\mathbf{u}\rangle}{\partial t}+\langle\mathbf{u}\rangle \cdot \nabla\langle\mathbf{u}\rangle\right)=-\nabla \cdot(\rho Q)+\rho \mathbf{g}-\nabla p+\nabla \cdot\langle\tau\rangle
$$

which contains a new term $-\nabla \cdot(\rho Q)$, where $Q$ is a tensor, the Reynolds stress tensor, consisting of the products of different velocity components, $Q_{i j}=\left\langle u_{i}^{\prime} u_{j}^{\prime}\right\rangle$. To understand the role of this term we may write down the conservation law for angular momentum

$$
\frac{\partial}{\partial t}\left(\rho r^{2} \sin ^{2} \theta \Omega\right)+\nabla \cdot\left(\rho r^{2} \sin ^{2} \theta \Omega\langle\mathbf{u}\rangle+\rho r \sin \theta\left\langle u_{\phi}^{\prime} \mathbf{u}^{\prime}\right\rangle\right)=0 .
$$

Thus, the angular momentum is transported not only by meridional circulation, but also by Reynolds stresses.

As we see, $Q$ appears inside a divergence as does the viscosity tensor $\tau$. Classically, the simplest approximation for $Q$ is the Boussinesq-ansatz $Q_{i j}=-\nu_{T}\left(\left\langle u_{i, j}\right\rangle+\right.$ $\left.\left\langle u_{j, i}\right\rangle\right)$. It is then identical to the viscosity tensor, except $\nu$ is replaced with an effective viscosity $\nu_{T}$, the "turbulent" or "eddy" viscosity. However, this approximation 


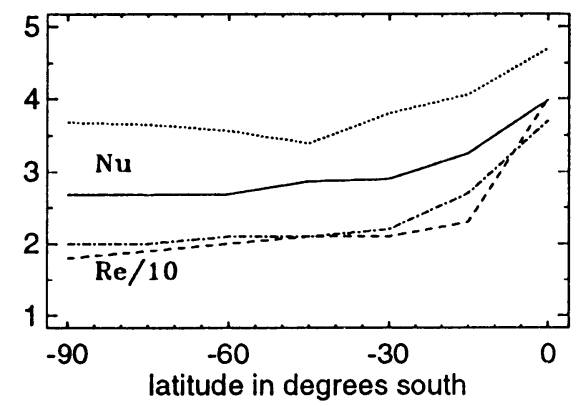

Fig. 1. Nusselt and Reynolds numbers vs. latitude. The lower curves represent the case for $\mathrm{Ra}=30000$ (solid line for $\mathrm{Nu}$ and dashed for $\mathrm{Re}$ ), and the upper curves are for $\mathrm{Ra}=60000$.

gives the wrong sign for $Q_{\theta \phi}$ compared with the observations of the solar surface. Moreover, physically we need something nondiffusive that maintains the existing gradients of the angular velocity and the anisotropy of the turbulence. Rüdiger (1989) used general arguments, taking as preferred directions those of gravity and angular velocity, and was able to derive the latitude-dependence of the Reynolds stress components in his $\Lambda$-theory.

$$
\begin{aligned}
& Q_{i j}=\Lambda_{i j k} \Omega_{k}-N_{i j k l} \partial\left\langle u_{k}\right\rangle / \partial x_{l}+\ldots, \\
& \frac{Q_{r \phi}}{\nu_{T}}=\left(-\frac{r}{\Omega} \frac{\partial \Omega}{\partial r}+V^{(0)}+V^{(1)} \sin ^{2} \theta+V^{(2)} \sin ^{4} \theta\right) \sin \theta \Omega, \\
& \frac{Q_{\theta \phi}}{\nu_{T}}=\left(-\frac{1}{\Omega} \frac{\partial \Omega}{\partial \theta}+H^{(1)} \cos \theta \sin \theta+H^{(2)} \cos \theta \sin ^{3} \theta\right) \sin \theta \Omega .
\end{aligned}
$$

The coefficients $V^{(0)}, \ldots, H^{(2)}$ have been derived from simple turbulence models using the First-Order-Smoothing approach (e.g. Rüdiger 1989). Given the limitations of this approach it is useful to determine these coefficients independently using three-dimensional simulations of convection.

\section{Results of the Simulations}

\subsection{Case I: Simulations with one unstable layer}

We performed a number of simulations, placing the box at eleven different latitudes in the southern hemisphere. An interesting tendency is seen in the behaviour of convective motions: convection is clearly stronger in the equatorial regions than at the pole, which results in larger Nusselt and Reynolds numbers (fig. 1). Gilman (1977) also finds that convection is most favoured in equatorial regions and Busse \& Cuong (1977) note that at the poles and equator pressure gradients can balance the Coriolis force and thus help to neutralize the stabilizing effect of rotation, but at the poles the Coriolis force has only horizontal components and the enhanced gradients increase dissipation there. 


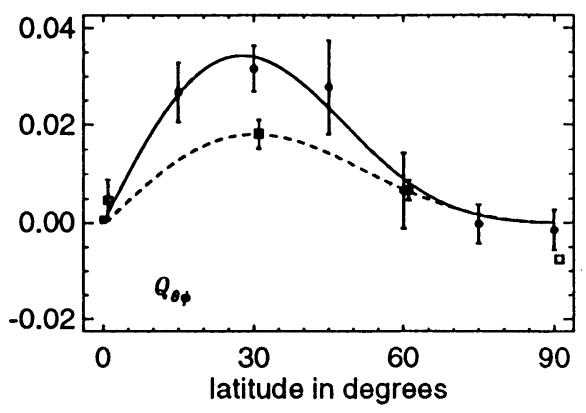

Fig. 2. Derived values for the horizontal Reynolds stress $Q_{\theta \phi}$ at different latitudes. The circles denote results from case I and boxes from case II. $w_{1}$ is 0.182 and 0.107 , and $w_{2}$ is 1.02 and 0.163 in those cases, respectively (see eq. 7).

According to our simulations the horizontal Reynolds stress, $Q_{\theta \phi}$, is negative everywhere in the southern hemisphere and changes sign at the equator, in agreement with the expected symmetry requirements. This was also checked by one run at 30 degrees north. In fig. 2 we give this correlation translated to the northern hemisphere, having therefore positive sign. We can fit the latitudinal profile of $Q_{\theta \phi}$ to the models of differential rotation (e.g. Tuominen \& Rüdiger, 1989)

$$
Q_{\theta \phi}=\nu_{T} \Omega_{*}\left(w_{1}+w_{2} \sin ^{2} \theta\right) \cos \theta \sin ^{2} \theta,
$$

where we have adopted the rotation law from the solar surface, $\Omega=A+B \cos ^{2} \theta+$ $C \cos ^{4} \theta$, thus. being able to present $Q_{\theta \phi}$ with just two unknown coefficients, $w_{1}$ and $w_{2}$ (see fig. 7 in Pulkkinen et al. 1993). This fit agrees well with analysis of the Greenwich observations. For comparisons between the simulations and other sources see Pulkkinen et al. (1993).

Because of the stress-free boundary conditions of our model, the correlation $Q_{r \phi}$ vanishes at the upper and lower boundaries. Therefore we choose to evaluate it in the mid-layer of the box. Fig. 3 shows this correlation as a function of latitude. No observations of $Q_{r \phi}$ are available for comparison. $Q_{r \phi}$ is clearly negative and also has the same sign in the northern hemisphere, because the radial and latitudinal flows do not change their sign at the equator.

\subsection{Case II: Simulations With two layers, upper unstable and lower STABLE}

The significance of this simulation is the possibility to study a more turbulent situation than the one in Case I, and also the effects caused by a boundary zone between the stable and unstable layers. The stratification is also much greater (about 20). The lower layer is convectively stable and the upper one unstable. Four runs at latitudes $0,30,60$, and 90 degrees north are considered.

The latitude-dependence of the strength of convection that was present in case I seems to have mostly disappeared, but not because of the stable layer, which is found to have little effect on the nature of convection. In this more turbulent case the velocity is generally more isotropic, and the horizontal and vertical components 


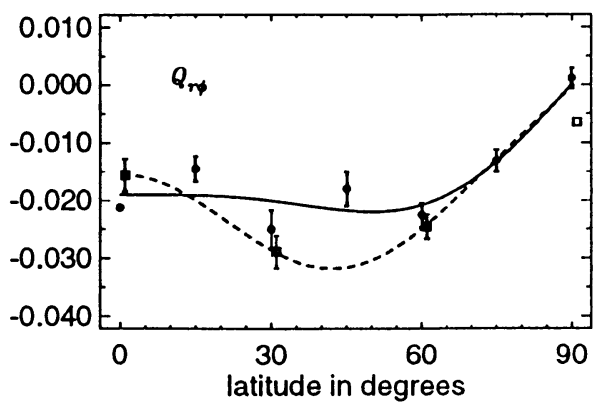

Fig. 3. Derived values for the Reynolds stress $Q_{r \phi}$. The $V$-coefficients (eq. 5) are -0.580 , 0.661 , and -0.279 in case $I$, and $-0.224,-0.100$, and 0.250 in case II, respectively.

are more or less equal. Only close to the equator the horizontal velocity components are somewhat larger than the vertical one. Here, $\left\langle u_{\phi}^{2}\right\rangle$ is largest in the upper part of the unstable layer. Nevertheless, the latitude-dependence of $Q_{\theta \phi}$ is qualitatively similar to that in case I (see fig. 2), but is about a factor of two smaller. In the lower layer, which is convectively stable, $Q_{\theta \phi}$ has the opposite sign, but much smaller magnitude than in the upper parts. As a whole, these results for $Q_{\theta \phi}$ are consistent with the ones of case I, and it appears that the qualitative behaviour of the horizontal Reynolds stress only depends weakly on the strength of turbulence. $Q_{r \phi}$ shows a somewhat stronger latitudinal variation than in case I; see fig. 3 . In the unstable layer $Q_{r \phi}$ behaves quite similarly than in case I, but the vertical flow penetrates into the stable layer causing a non-zero tail for this correlation there. At the equator $Q_{r \phi}$ goes to zero in the middle of the upper layer which is possibly related to the enhanced anisotropy there.

\section{Final remarks}

The behaviour of the Reynolds stress seems to be quite well simulated by computer models. The role of the Coriolis force is essential and therefore, a local model like the one presented here is valid for describing the latitude-dependence of the Reynolds stresses. However, other quantities such as the strength of convection and meridional flow may require a global model, or at least more detailed study with the present model, accepting its limitations.

\section{References}

Busse, F.H., Cuong, P.G.: 1977, Geophys. Astrophys. Fluid Dyn. 8, 17

Dziembowski, W.A., Goode, P.R., Libbrecht, K.G.: 1989, Astrophys. J. 337, L53

Gilman, P.A.: 1977, Geophys. Astrophys. Fluid Dyn. 8, 93

Nordlund, A., Stein, R.F.: 1989, in Solar and Stellar Granulation, ed(s)., R. Rutten and G. Severino, Kluwer Acad. Publ., 453

Pulkkinen, P., Tuominen, I., Brandenburg, A., Nordlund, Å., Stein, R.F.: 1993, Astron. Astrophys. 267, 265

Rüdiger, G.: 1989, Differential Rotation and Stellar Convection: Sun and Solar-type Stars, Akademie-Verlag, Berlin \& Gordon and Breach, New York

Tuominen, I., Rüdiger, G.: 1989, Astron. Astrophys. 217, 217 